\title{
Development of Laparoscopic Cholecystectomy Simulator Based on Unity Game Engine
}

\author{
Jinglu Zhang \\ National Center for Computer \\ Animation \\ Bournemouth University \\ zhangj@bournemouth.ac.uk \\ Yinyu Nie \\ National Center for Computer \\ Animation \\ Bournemouth University \\ ynie@bournemouth.ac.uk
}

\author{
Yao Lyu \\ National Center for Computer \\ Animation \\ Bournemouth University \\ ylyu@bournemouth.ac.uk \\ Xiaosong Yang \\ National Center for Computer \\ Animation \\ Bournemouth University \\ xyang@bournemouth.ac.uk \\ Jian Chang* \\ National Center for Computer \\ Animation \\ Bournemouth University \\ jchang@bournemouth.ac.uk
}

\author{
Yukun Wang \\ National Center for Computer \\ Animation \\ Bournemouth University \\ i7254611@bournemouth.ac.uk \\ Jianjun Zhang \\ National Center for Computer \\ Animation \\ Bournemouth University \\ jzhang@bournemouth.ac.uk
}

\begin{abstract}
Fast development of the minimally invasive surgery (MIS) technology demands extra surgical skills training to meet advanced technological challenges. However, massive capital expenditures and ethical issues with safety considerations exist in traditional surgical training methods (e.g. using cadavers or animals). Those limitations turn Virtual Reality (VR) surgery simulation into a plausible alternative to provide a safe and repeatable virtual training environment. In this paper, we design and develop a game engine based laparoscopic cholecystectomy training simulator for surgeons to understand the surgery procedure and practice their surgical skills as well as decision making skills. Our design leverages physical simulation and haptic force feedback to offer trainees a realistic visual and tactile experience, respectively. We explore the possibility of using game engine rather than developing from scratch to build the surgical simulator. Based on the results and user feedbacks from a pilot experiment, we conclude that game engine is a viable option for creating a cost-effective, flexible and highly interactive virtual surgery training platform for pedagogical purpose, which can shorten the development time with some compromise in functionality.
\end{abstract}

${ }^{*}$ Corresponding Author

Permission to make digital or hard copies of all or part of this work for personal or classroom use is granted without fee provided that copies are not made or distributed for profit or commercial advantage and that copies bear this notice and the full citation on the first page. Copyrights for components of this work owned by others than ACM must be honored. Abstracting with credit is permitted. To copy otherwise, or republish, to post on servers or to redistribute to lists, requires prior specific permission and/or a fee. Request permissions from permissions@acm.org.

CVMP '18, December 13-14, 2018, London, United Kingdom

(c) 2018 Association for Computing Machinery.

ACM ISBN 978-1-4503-6058-6/18/12 . \$ \$15.00

https://doi.org/10.1145/3278471.3278474

\section{CCS CONCEPTS}

- Human-centered computing $\rightarrow$ Virtual reality; Haptic devices; • Computing methodologies $\rightarrow$ Physical simulation;

\section{KEYWORDS}

Surgery simulation, cholecystectomy, game-based training, virtual surgery, modeling

\section{ACM Reference Format:}

Jinglu Zhang, Yao Lyu, Yukun Wang, Yinyu Nie, Xiaosong Yang, Jianjun Zhang, and Jian Chang. 2018. Development of Laparoscopic Cholecystectomy Simulator Based on Unity Game Engine. In Proceedings of CVMP '18: European Conference on Visual Media Production (CVMP '18). ACM, New York, NY, USA, 9 pages. https://doi.org/10.1145/3278471.3278474

\section{INTRODUCTION}

Cholecystectomy, a surgical procedure to remove the gallbladder, is one of the most common operating room surgeries. With the explosion of related minimally invasive surgery (MIS) techniques, cholecystectomy can be operated with the assistance of a video camera and several laparoscopic instruments. Benefits of laparoscopic cholecystectomy include few post-operative complications, small incisions on the skin, and a relative shorter recovery period [Suzuki et al. 2000]. However, the limited viewing angle and restricted operating space during laparoscopic cholecystectomy often cause undesirable complications (such as bile duct leak or injury). Therefore, it is indispensable for surgeons to acquire pertinent surgical skills prior to a real surgery.

Traditionally, using cadavers or animals for training faces many ethical issues. Training by supervised experienced surgeons is a feasible solution, but it is at the cost of the huge expenditure and a long training period. Fortunately, virtual reality (VR) simulator has gradually become an effective approach [Alaker et al. 2016] to teach and assess surgical skills outside operation rooms. Medical 
students and surgeons can practice their professional skills along with the transferable skills such as team-working and emergency reaction using this advanced technique. Many research works have proven the correlation between game-based training and the improvement of surgical skills [Andreatta et al. 2010; Creutzfeldt et al. 2010; Knight et al. 2010; Kurenov et al. 2009; Rosser et al. 2007] Specifically, advantages of the surgical simulator include:

(1) offering a secure and efficient environment for surgeons to deeply understand the whole surgery process

(2) enabling surgeons to practice two essential skills for MIS, namely eye-hand coordination and the ability to execute 3D actions using a $2 \mathrm{D}$ screen as a guide

(3) allowing trainees to practice the challenging and significant procedures repeatedly and save the training cost

It is complicated to model and simulate a typical laporoscopic cholecystectomy scene. The simulation of multiple organs and tissues, the interaction between tools and tissues, and feedbacks from both visual and tactile are all necessary components. Developing and integrating all these functional components independently is time and resources consuming. Unity [Goldstone 2009] is a cross-platform modern game engine which has a great support of advanced audio and visual effects. Its integrated development environment, easy profiler, on-shelf tools and modules make Unity stable and highly productive.

In this study, we design and develop a laparoscopic cholecystectomy simulator using Unity Game Engine. Our surgical simulator provides trainees an effective platform to practice surgery procedures with realistic visual and haptic feedbacks (shown in Fig. 1 and Fig. 2). In our design, the organ and soft tissue simulation are based on uflex, a unity plugin originated from NVidia Flex[Korzeniowski 2016], and the haptic feedback is in reference to Unity 5 Haptic Plugin for Geomagic OpenHaptics 3.3 [Poyade et al. 014 ]. There are mainly two contributions of our work:

(1) Developing an interactive laparoscopic cholecystectomy simulator for surgeons to enhance their surgical skills

(2) Exploring the use of game engine for novel development of a surgical simulator to achieve efficient and cost-effective solutions

The remaining of this paper is organized as follows. Section 2 reviews previous works in medical education and training, laparoscopic simulator and soft body deformation modeling. Detailed game objective, architecture, and target users are given in section 3. Section 4 explains our real time physics simulation algorithms in depth, and section 5 gives the system evaluation and feedbacks. Finally, section 6 concludes the paper.

\section{RELATED WORK}

Game like medical education and surgical skills training with different objectives have been proven to be valuable where users can improve their related skills and experience various circumstances that are impractical in the real world due to the safety, cost, or other reasons [Susi et al. 2007]. James et al. presented that surgeons who had experienced selected representative video games (Super Monkey Ball 2, Star Wars Racer Revenge, and Silent Scope) performed better in laparoscopic handling and made fewer errors [Rosser et al.

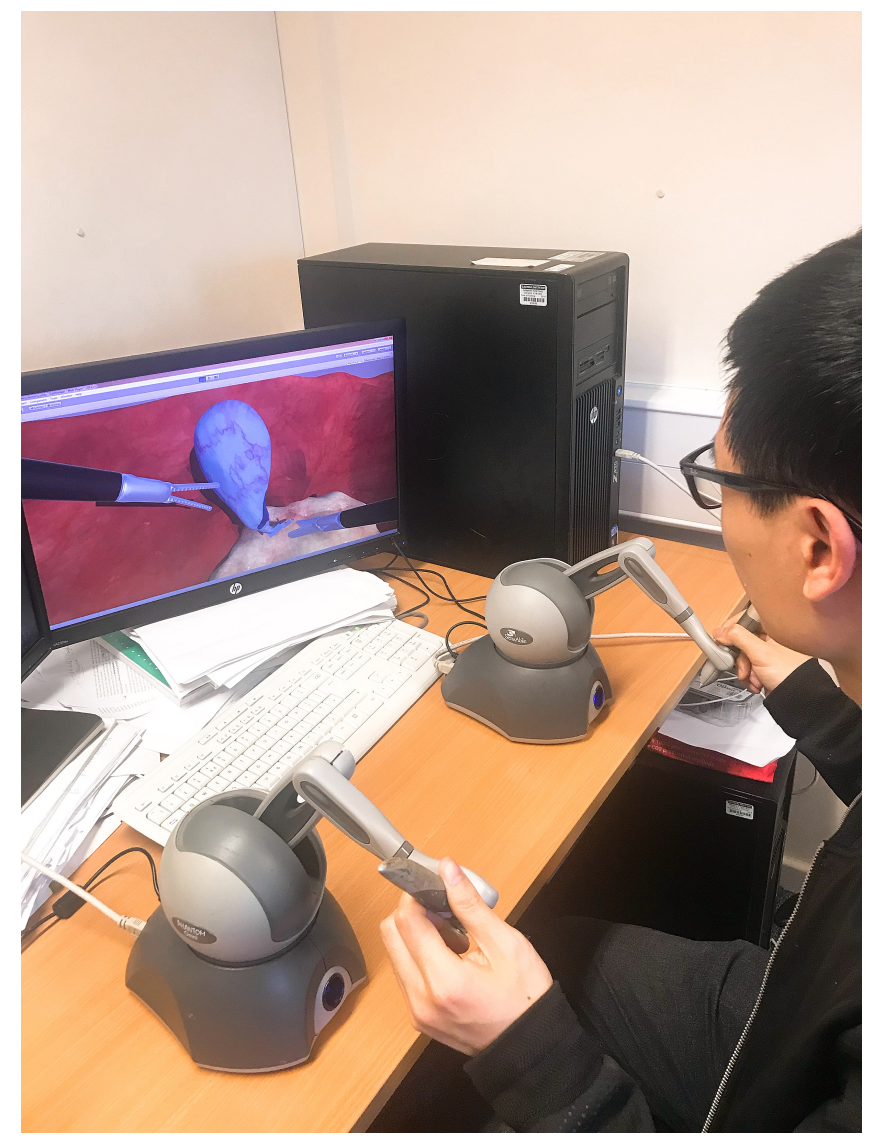

Figure 1: Laparoscopic cholecystectomy simulator working environment.

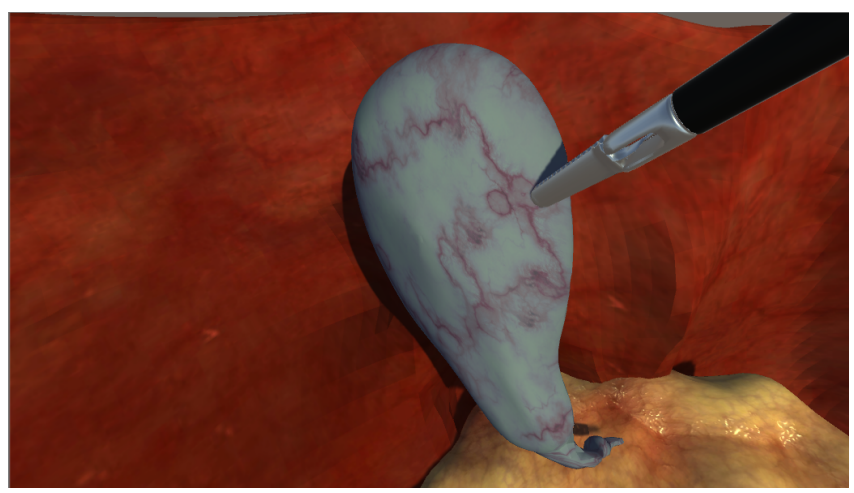

Figure 2: Screenshot from cholecystectomy simulator.

2007]. Another interesting experiment [Knight et al. 2010] compared the tagging accuracy between participants who had undertaken the game, Triage Trainer (Blitz Games Studios, Leamington Spa, Uk) training and who had taken traditional card-sort exercise. The Triage Trainer group was more likely to triage precisely in casualties than the card-sort group. Game like training was also used for team training in emergency crisis management [Andreatta et al. 
2010; Kurenov et al. 2009], daily clinical tasks for junior doctors, and cardiopulmonary resuscitation [Creutzfeldt et al. 2010], etc. In this research, we focus on the laparoscopic surgical skills trained via the surgical simulator.

Simulators for surgical skills training can be categorized into two types: low fidelity and high fidelity. Evaluation criteria of fidelity are decided by the extent of visual and tactile realism and system interactivity. Synthetic models [Hammoud et al. 2008] and Video Box Trainer belong to low-fidelity simulators. Synthetic models are static bench models such as tissue models for fascia closure and knot-tying training. Video Box Trainer [Fried et al. 2004] takes advantage of real laparoscopic instruments as well as the camera and video monitors to simulate surgery procedures. Although low fidelity simulators are cost-efficient, easy to make and portable, they usually sacrifice the realism and only offer a single surgical skill training rather than a whole process.

Virtual reality surgery training is a type of high fidelity surgical simulation. This type of technology satisfies the requirements of taking 2D screen as a guide to performing 3D surgery with realistic visual and tactile feedback while also has the ability to monitor and record the training progress. Most of the existing surgical simulators either reinvent all components independently [Pan et al. 2015; Qian et al. 2015] or build from an extendable framework such as SOFA [Kim et al. 2015] and GiPSi [Cavusoglu et al. 2006]. Whereas building medical educational or training systems upon game engines is not fully explored. Few research works [Marks et al. 2007a,b] have discussed the possibilities and advantages of game engine based surgery simulators. In order to bring more understanding in this direction, we use a robust multipurpose game engine Unity to build our simulation architecture. We also evaluate and compare the pros and cons between the game engine and other simulation methods for surgical training.

Immersive surgery simulation requires accurate physical behaviors, the precision of soft tissue deformation enormously affects the sense of reality of the whole framework [Gallagher et al. 2005; Zhang et al. 2017]. Three types of simulation approaches have been widely applied nowadays.

- Force-based approaches: Mass-spring [Baraff and Witkin 1998; Bouaziz et al. 2014; Liu et al. 2013] system consists of sets of point masses connected by spring dampers. It is a simple and efficient scheme which takes Hooke's Law as the theoretical basis. But it is hard to tune the spring constants to get a desired behavior and usually causes the overshooting problem.

- Structural-based approaches: Unlike the mass-spring system which discretize an object into finite number of point masses, finite element method (FEM) [Zienkiewicz and Taylor 2005] is based on continuum mechanics theory. It is capable of handling accurate physical behaviors for different types of elastic and non-elastic material. However, the model complexity associates the technique with difficult initial settings and high computational cost [Sifakis and Barbic 2012].

- Position Based Dynamics (PBD) [Müller et al. 2007]: PBD is a method which works on positions directly in each simulation step to resolve constraints. It is fast, stable, and controllable which makes the simulation process highly efficient and functionally suitable for the interactive environment [Bender et al. 2014]. Despite the fact that PBD is not physically accurate, it achieves real time surgical simulation which is still visually plausible. We applied the PBD solver with different constraints for our physics simulation as a test prototype.

\section{SYSTEM INFRASTRUCTURE}

\subsection{Procedures and Challenges in Laparoscopic Cholecystectomy}

Laparoscopic Cholecystectomy has gradually replaced the open surgery to become the major modality of treating gallstones, gallbladder carcinoma, trauma and porcelain gallbladder. Four primary steps are involved in the surgery as shown in Fig. 3: a) Position the patient correctly and insert the trocars and camera through three labeled minimal ports; b) Identify the position of the gallbladder, and then dissect the tissue to find the cystic duct and cystic artery; c) Clip and ligate the cystic duct and cystic artery structure; and d) Tease away adhesions between the gallbladder and the liver, put the gallbladder into a pre-prepared bag and drag it out. In our training system, we focused on the core steps b), c), and d), as a) can be conducted in a separate training session.

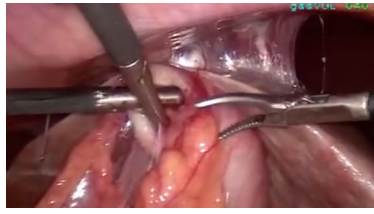

(a) Initial view

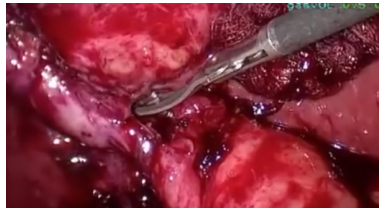

(c) Clipping and cut

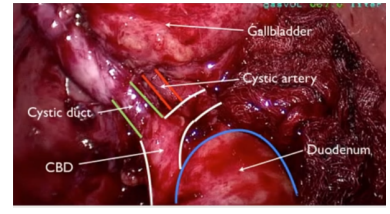

(b) Dissect Calot's Triangle

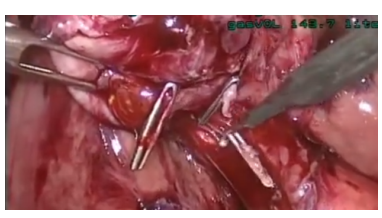

(d) Teasing away the gallbladder
Figure 3: Surgery process. Image by School of Surgery. [Jones 2014]

During the intra-operative process, some challenges and difficulties require a particular attention, especially for a novice:

- Identifying the Calot's triangle properly is the prerequisite to safely remove the gallbladder. Calot's triangle, shown in Fig. 4 , is the most important anatomy structure in laparoscopic cholecystectomy. It helps the surgeon clearly identify the relative position among the cystic duct, common hepatic duct, and inferior border of the liver.

- Always clipping the cystic duct and cystic artery first and then cutting them to prevent uncontrollable bleeding or bile leaking.

- Gallbladder closely connects with the liver. Correctly separating adhesions between them prevents the harm to the liver.

- In laparoscopic surgery, a surgeon operates $36-39 \mathrm{~cm}$ long instruments through a tiny entry point, such that the unskilled 
and careless manipulation is possible to damage intestines or main blood vessels.

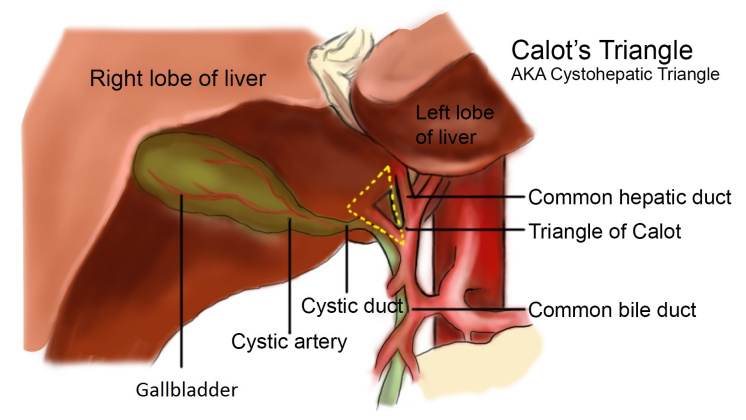

Figure 4: Calot's Triangle. [Suzuki et al. 2000]

\subsection{Objectives and System Design}

This laparoscopic cholecystectomy simulator is designed and developed for medical students and junior doctors to train their surgical skills in a safe, repeatable and cost-effective manner. Medical students can thoroughly understand the surgery process and practice essential skills before a hand-on operation on a real patient. As for junior doctors lacking practical experiences, they are able to draw lessons from their weakness and practice repeatedly to further enhance operation skills. Laparoscopic experts are involved to monitor the progress of students and juniors to give valuable feedbacks and guidances during the training.

Typically, a surgery training simulator includes a rendering module for displaying 3D models and user interface, a physics simulation module for collision detection, rigid body, soft tissue, and smog/fluids simulation, and an event handling module for input/output. In our development, to exclude unnecessary complexity, we only prototype the anatomical structure for a normal patient without lesions. The patient specific mode will be the future work, which would be of better use to benefit surgical planning. Concretely, we design the game module as below (Fig. 5 shows four crucial steps) :

(1) A video [Jones 2014] illustrates the process of labeling the patient and inserting trocars through abdomen.

(2) Viewpoint selection is important on account of the limited vision field of minimally invasive surgery. Users are able to adjust the camera to get the optimal viewpoint by keyboard.

(3) After identifying correlated anatomical structures, trainees need to clip three staples in correct positions on the cystic duct and ligate the gallbladder.

(4) With the help of two haptic devices, trainees are required to separate the adhesion between the gallbladder and the liver

(5) The remaining task is rather simple, so we use another video clip to present the procedure of putting the gallbladder into a pre-prepared bag and dragging it out.

\section{PHYSICAL SIMULATION}

NVIDIA Flex is a particle-based simulation library for real time visual effects. In Flex, everything is modeled as a system of particles connected by different constraints, which is broadly based on PBD and Unified Particle Physics [Macklin et al. 2014]. NVIDIA FleX does not support the Unity Game Engine directly. Instead uFlex, a Unity asset integrated low-level Flex native library, is applied in our development. The core idea is to solve a non-linear system sequentially with equality and inequality constraints in different time steps:

$$
\begin{gathered}
C_{i}(\mathbf{p}+\Delta \mathbf{p})=0, \quad i=1, \ldots n \\
C_{j}(\mathbf{p}+\Delta \mathbf{p})>=0, \quad j=1, \ldots n
\end{gathered}
$$

where $\mathbf{p}=\left[\mathbf{p}_{1}, \mathbf{p}_{2}, \ldots, \mathbf{p}_{N}\right]^{T}$ is the vector of particle positions. The unified representation allows modeling different materials and interactions between various types of models, namely rigid body, soft body, cloth, and fluids in a fully efficient and flexible manner. There are mainly two types of the soft tissue associated with laparoscopic cholecystectomy: volumetric soft tissue and surface deformable tissue. We explain their modeling methods as follows.

\subsection{Volumetric Soft Body Simulation}

Liver, gallbladder and fat tissues are regarded as 3D volumetric deformable objects in cholecystectomy, since they keep their intrinsic shape most of the time. When instruments contact or manipulate the organ, only the contacted part deformed. Shape matching constraints of clusters are used to simulate soft bodies. The core idea of shape matching [Müller et al. 2005] can be described as: Attracting initial point sets $\mathbf{x}_{i}^{0}$ towards the "goal" positions $\mathbf{p}_{i}$ by finding the global optimal transformation and rotation matrix against the current state. That is to minimize:

$$
\sum_{i} w_{i}\left(\mathbf{R}\left(\mathbf{x}_{i}^{0}-\mathbf{t}_{0}\right)+\mathbf{t}-\mathbf{p}_{i}\right)^{2}
$$

where weights $\left\{\mathbf{w}_{i} \mid i=1, \ldots n\right\}$ in this case are mass, $\mathbf{R}$ is the rotation matrix, and $\mathbf{t}$ is the translation vector. The optimal translation vectors turn out to be the displacement of the center of mass for the shape, and the global rotation matrix is extracted from the global transformation matrix by polar decomposition. Finally, the goal potion can be calculated as:

$$
\mathbf{p}_{i}=\mathbf{R}\left(\mathbf{x}_{i}^{0}-\mathbf{x}_{c m}^{0}\right)+\mathbf{x}_{c m}
$$

where $\mathbf{x}_{c m}$ is the center of mass.

Throughout the simulation, a soft body consists of several clusters is shown in Fig. 6. Each particle belongs to one or more clusters with a weight between 0 and 1 , standing for the extent of a particle influenced by the corresponding cluster. The final deformation result is the overlapping and averaging of the constraints which defines in each cluster independently as described by the shape matching model. The more clusters in a soft body, the more elastic it will become. Shape matching constraint does not need the connectivity information, and the result is easy to compute, thus it is efficient for an interactive environment which compromises some physical accuracy. 


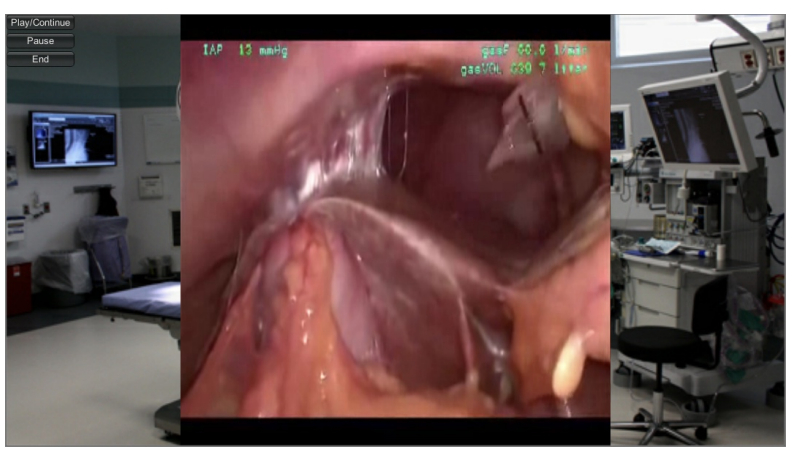

(a) Port insertion

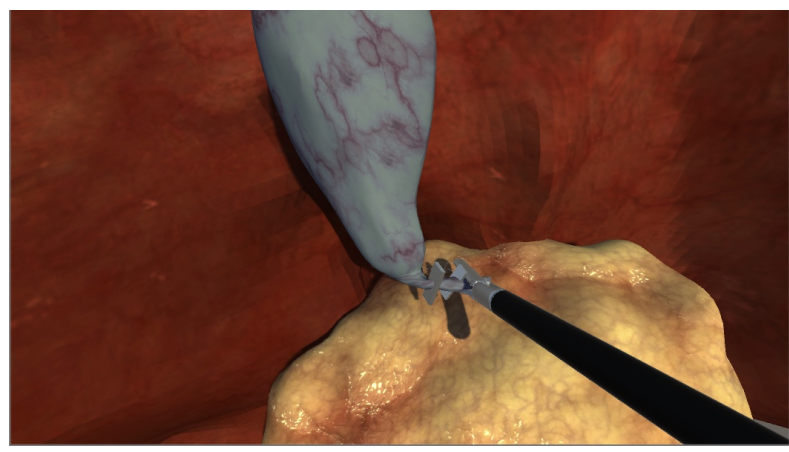

(c) Clipping and ligating the cystic duct

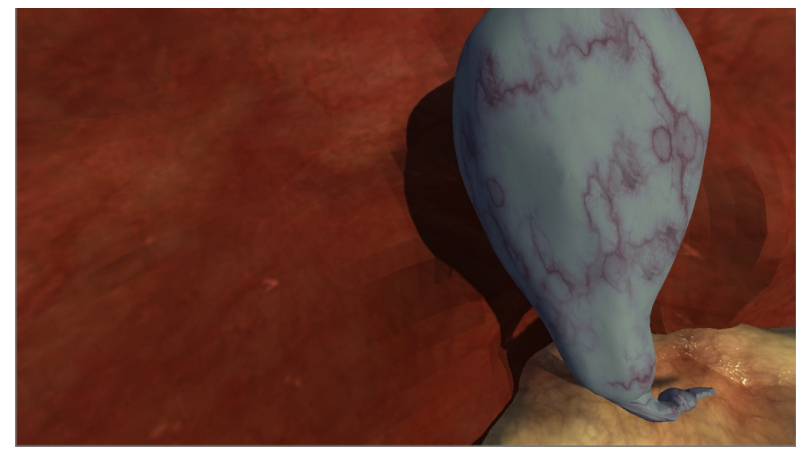

(b) Anatomical structure identification

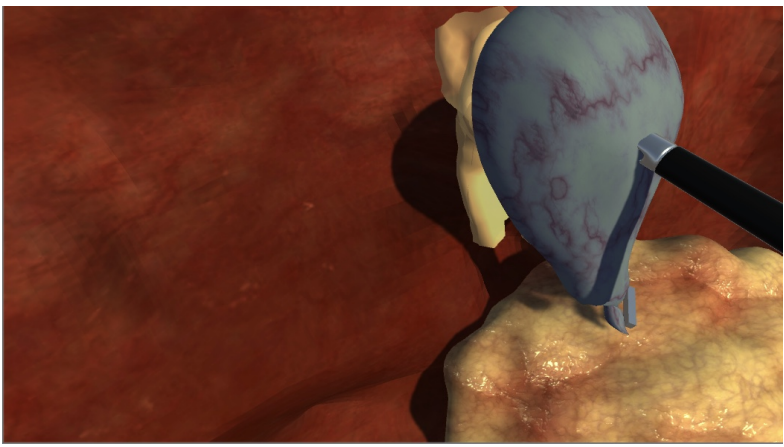

(d) Teasing away the gallbladder

Figure 5: Operation procedures.

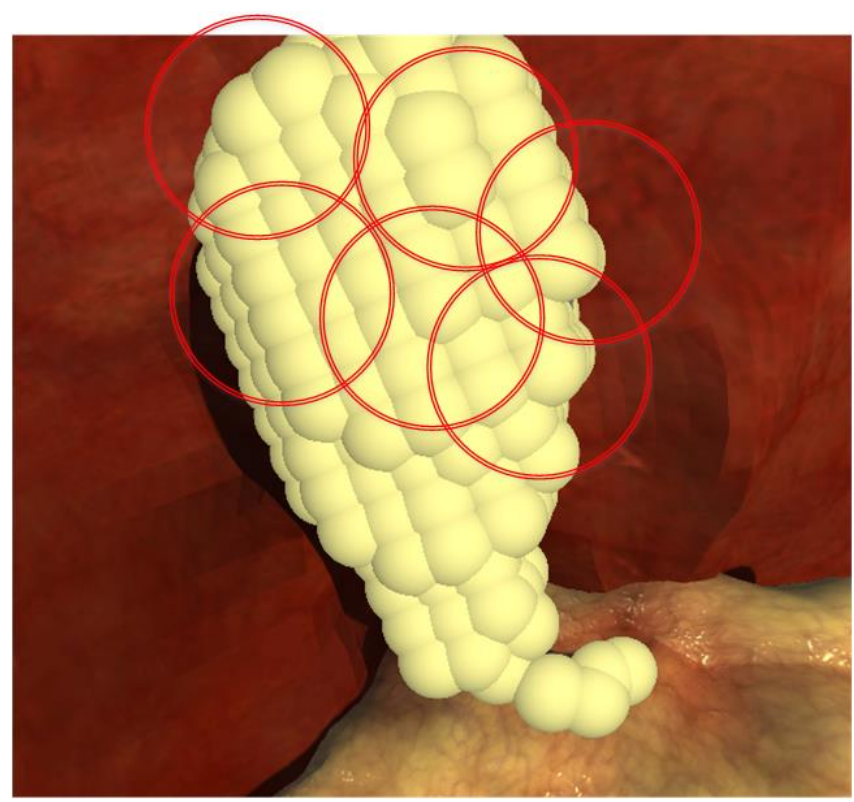

Figure 6: Clustering in shape matching.

\subsection{Surface Mesh Simulation}

We need to simulate the fascia tissue between the gallbladder and the liver in the dissection procedure. Given the extensible and thin features of the fascia, it is modeled using cloth simulation. The fascia is displayed as spring networks, in which one triangle handles the stretch and two adjacent triangles handle the bending constraint. For each edge, the stretching constraint function is:

$$
C_{\text {stretch }}\left(\mathbf{p}_{1}, \mathbf{p}_{2}\right)=\left|\mathbf{p}_{1}-\mathbf{p}_{2}\right|-l_{0}
$$

where $l_{0}$ is the rest length of the edge. As for two neighbor triangles $\left(\mathbf{p}_{1}, \mathbf{p}_{3}, \mathbf{p}_{2}\right)$ and $\left(\mathbf{p}_{1}, \mathbf{p}_{2}, \mathbf{p}_{4}\right)$, the bending constraint is generated as:

$$
C_{\text {bend }}\left(\mathbf{p}_{1}, \mathbf{p}_{2}, \mathbf{p}_{3}, \mathbf{p}_{4}\right)=\arccos \left(\mathbf{n}_{1} \cdot \mathbf{n}_{2}\right)-\varphi_{0}
$$

where $\mathbf{n}_{1}$ and $\mathbf{n}_{2}$ are the normal vector of two triangles and $\varphi_{0}$ is the dihedral angle between two triangles in the rest pose. The overall goal is to minimize total energy defined as:

$$
E=\sum\left(k_{\text {stretch }} C_{\text {stretch }}^{2}+k_{\text {bend }} C_{\text {bend }}^{2}\right)
$$

where $k_{\text {stretch }}$ and $k_{\text {bend }}$ are global stiffness parameters provided by the user.

However, the fascia simulation is not visually plausible in our test, because fascia is not a single layer of smooth structure as the cloth. While splitting the gallbladder from the liver in our simulation, the fascia just looks like a piece of cloth with explicit triangle mesh in the torn partition as shown in Fig. 7(a). We design an adhesive constraint to simulate the breakable fascia to enhance the visual effects. Because uFlex can only access to the basic-level library of NVIDIA Flex, it cannot simulate the sticky effects. We tested the algorithm in Houdini, and we will integrate the adhesive constraint into system in the future work. As the result, the breakable fascia is visually satisfying as shown in Fig. 7(b). 


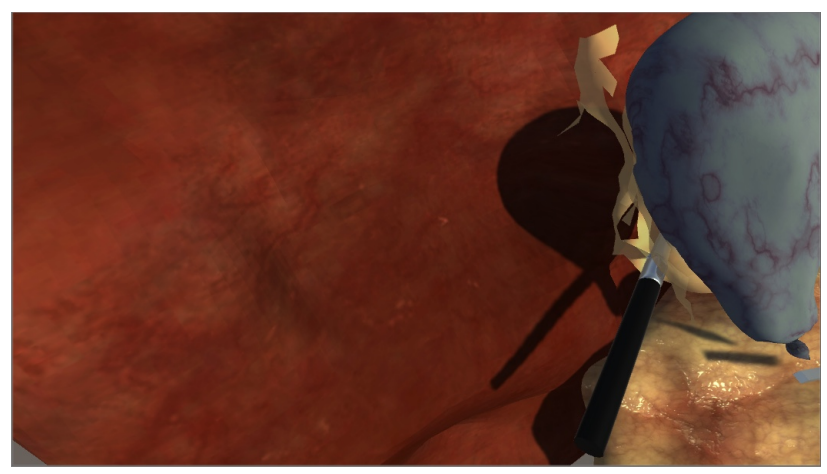

(a) Artifact in fascia simulation

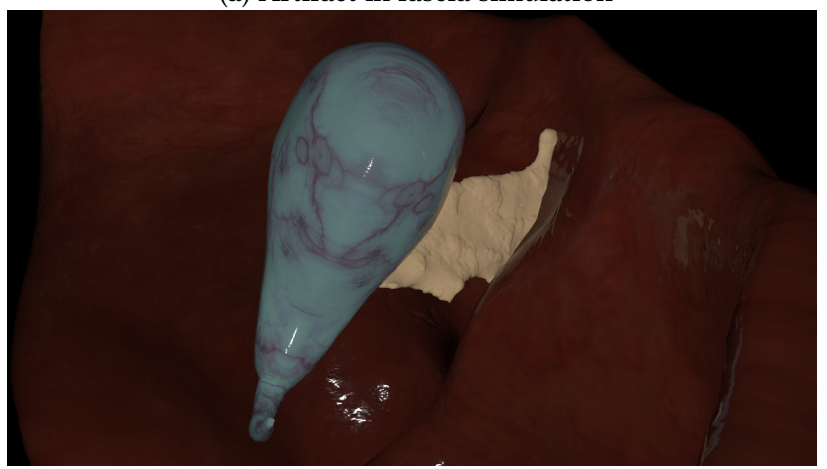

(b) Adhesive constraint for the fascia

Figure 7: Comparison between original and improved results.

In cloth simulation, fascia tissue is represented as connective triangle mesh. While in our new algorithm, we simply use lines and the distance constraint to model the fascia. Initially, we randomly generate several points on the surface of the gallbladder and the liver and connect them with lines. In each connection, few points are randomly scattered and marked as breakable group. At the same time, we assign a rest length and a very large stiffness parameter to each line. Finally when we drag the gallbladder, if the distance between two organs exceeds a given break threshold, the stiffness parameter of random breakable points will be reset to a very small number such that any force can break the connections. In such way, the connection between the gallbladder and the liver no longer looks like a piece of papery cloth with apparent triangle mesh. Instead, it presents a sticky behavior of the fascia when we drag the gallbladder away.

\subsection{Haptic Rendering}

A system requires at least 500-1000 $\mathrm{HZ}$ for smooth haptic rendering [Booth et al. 2003], in which $25-30 \mathrm{~Hz}$ for visual rendering in order to provide users realistic feedbacks. Haptic feedback is able to enhance both cognitive ability by offering continuous movement sensory and kinesthetic sense by differentiating different inner structures. Phantom Omni is a feasible and affordable haptic device for virtual-real interaction. It takes the $6 \mathrm{DOF}$ input and generates forces to constrain the 3 DOF output in a high sensory frequency. Haptic rendering in Unity enables the user to control haptic effectors as laparoscopic instruments to interact with organs and tissues.

Figure 8 shows the interactive work flow of the haptic device. At the beginning, we predefine the haptic workspace dimensions[Poyade et al. 014 ]. Within the workspace, meshes and transformation matrices of haptic geometries (organs and tissues) are set into haptic frame. During the training process, the position of the haptic proxy (i.e. the graphic representation of the haptic device in the screen, in our case is the position and orientation of the laparoscopic instrument model) keeps updating. If no collisions happen between instruments and the organ, the proxy position and the device position will stay the same. But if the proxy get contact with the haptic objects, the proxy position will be assigned to the device position. According to the position and the collided particle ID information, the simulation algorithm calculates the deformation and gives visual feedbacks to the screen and force feedbacks to the user hand.

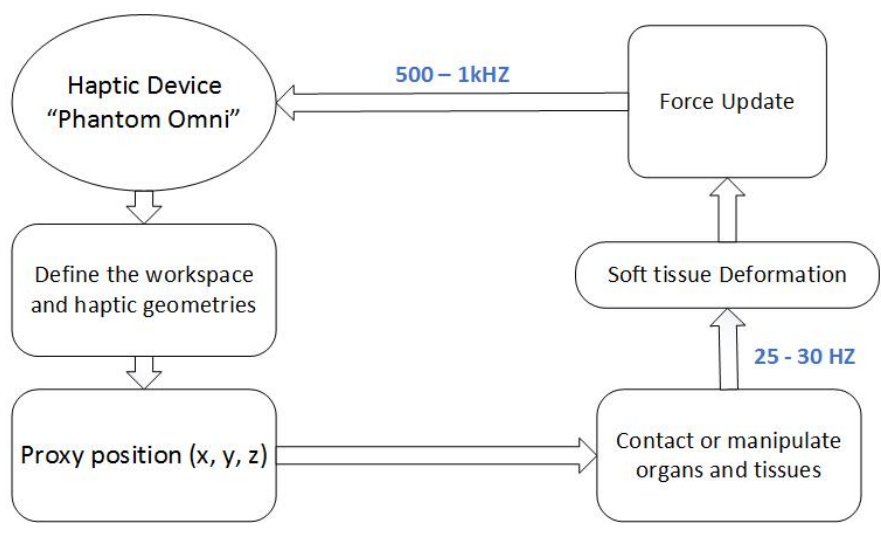

Figure 8: Haptic device workflow.

\section{EVALUATION AND FEEDBACK}

A PC with an Intel Xeon 5 CPU, a GeForceGTX 1080 graphics card, and two Phantom Omni devices is used to built this laparoscopic cholecystectomy simulator. The criterion-based quantitative assessment [Jackson et al. 2011] is employed to evaluate the overall capacity of the simulator from system performance and usability perspectives. Ten volunteer $\mathrm{PhD}$ students and five volunteers from general surgery department (including students and laparoscopic experts) participated in the test and gave their suggestions accordingly.

\subsection{System Evaluation}

There are five criteria chosen to evaluate the system performance: 1) ease of use, 2) interactivity, 3) visual realism, 4) freedom of movement and effectiveness, and 5) system stability. Ten volunteer $\mathrm{PhD}$ students are asked to grade each criterion from 1 to 5 , representing "Poor" to "Excellent". Figure 9 presents the average result of each criterion about the performance of our simulator. The system interactivity, freedom of movement and effectiveness and, ease 
of use receive the positive feedback with the score around four. All participants mentioned that haptic device with force feedback hugely improve the user experience. However, the visual realism and system stability need to be improved, due to the fact that the related FLEX solver is not physically accurate.

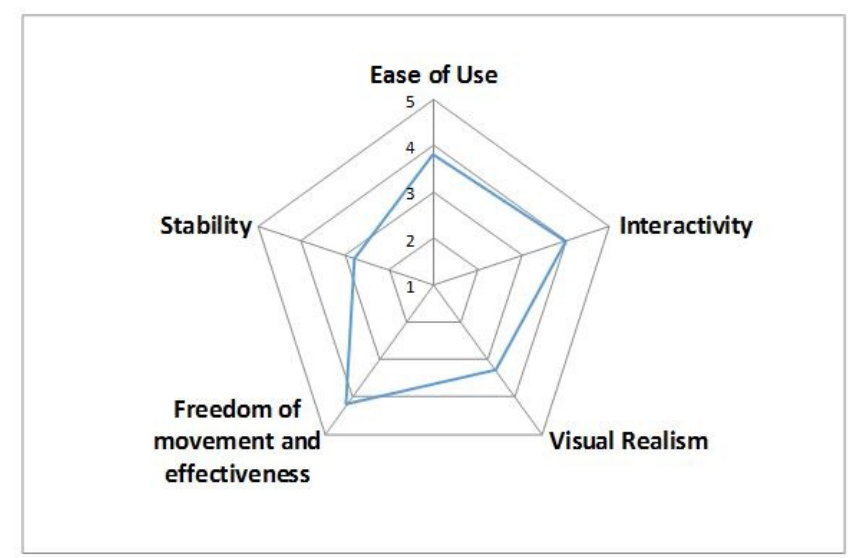

Figure 9: System performance evaluation. (1-Poor, 2-Fair, 3Average, 4-Good, 5-Excellent)

\subsection{Simulator Usability Evaluation}

Five criteria, namely 1) ease of use, 2) anatomy precision, 3) visual realism, 4) integrity of procedure, and 5) utility, are applied to assess the usability of the simulator. Evaluators from general surgery department are asked to grade each criterion from one to five, representing "Poor" to "Excellent". Figure 10 presents the average score of each criterion about the usability of our simulator. Due to the small sample numbers, such evaluation provided some guidance and further validation will be carried in the future work. The utility and ease of use reach the highest score around four, thus demonstrating the usefulness of VR surgical simulation. Nevertheless, there is still a space for improvement in anatomy precision, visual realism and integrity of procedure. Surgeons and medical students claim that as there are no professionals involved in our simulator design, some minimal details are neglected. For example, the inner structure of calot's triangle is more complex than the one we built. This defective anatomical representation has the potential to mislead trainers. In the future improvement, we will improve them with the guide from medical experts.

\subsection{Game Engine based Simulator}

Most of the existing simulators are either developed independently or based on some open-source framework, whereas we built the whole architecture upon Unity Game Engine. We have discussed our experimental results in a focus group of eight attendances from both computer scientists and laparoscopical surgeons. Comparing with other two types of simulators, we summarized advantages as well as limitations of using Game Engine for the surgical training.

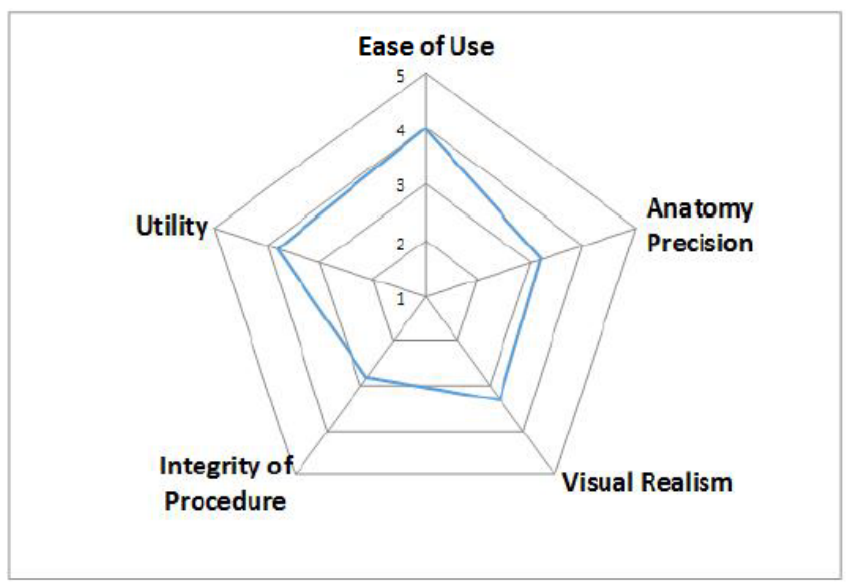

Figure 10: Simulator usability evaluation. (1-Poor, 2-Fair, 3Average, 4-Good, 5-Excellent)

\subsubsection{Advantages.}

- For the self-developed simulators, reinventing essential functional blocks (rendering, physic and event handling components) for a simulator is time-consuming and leads to huge development expenditure. In our case, we spent about four months for the whole development cycle, but it took one year or more for a simulator like [Qian et al. 2015] to achieve the similar function.

- Robust game engine architectures allow developers to concentrate more on the content rather than the implementation, thus could improve the user experience.

- Most of the simulator systems only focus on the technical training of the surgery process. However, other skills such as team collaborative and patient care are also very important for a qualified surgeon. The self-development simulators are not able to network with other simulators for collaborative tasks. None of them are audio supportive [Marks et al. 2007b]. Game engine has better support for network and audio.

\subsubsection{Limitations.}

- Game engines are primarily designed for game development. The physical simulation capability of virtual surgery still has a room for improvement. Unlike the ordinary soft body, the structure of human organs and soft tissues are very complex. The physics engine in Unity is not able to provide high fidelity laparoscopic features, e.g. cutting and multi-layer heterogeneous soft tissue simulation.

- It is a trade-off for a game engine to either sacrifice the precise animation for speed or emphasize on pleasant visual without real time interactive ability.

\subsection{Improvement Suggestions}

According to the evaluation results and suggestions from openended questionnaires, further improvements need to be achieved:

(1) Although the game engine has integrated a system development architecture for audio, rendering, event handling and networking, the visual realism of soft tissue deformation and 
the precision of collision detection needs to be improved. We will first integrate adhesive constraint simulation for fascia into our simulator.

(2) Several participants from medical institution indicate that we need more precise anatomy structures for calot's triangle. The success of laparoscopic cholecystectomy largely relies on whether surgeon could identify the anatomy structure properly.

(3) We will also add more interactive modules to improve the integrity and efficiency of the simulator. Currently, only one type of laparoscopic instrument is modelled in the simulator. Several instruments (retractor, electrodes, and anvil graspers) will be integrated, and users will be allowed to change the instruments for different tasks.

\section{CONCLUSION}

In this study, we have designed and developed a novel game engine based surgical simulator for laparoscopic cholecystectomy. Surgeons are able to train their surgical and decision making skills in this virtual environment before they encountering real patients. The VR surgical training allows trainees to operate the immersive cholecystectomy by using haptic devices with force feedbacks and a computer screen as the guide. The training environment is safe, cost-efficient, and repeatable. The user evaluation results demonstrate that the simulator is easy to use and interactive.

Currently, we only receive feedbacks from few laparoscopic surgeons and medical students. Before it can be validated as a surgery training tool, we need to further improve the evaluation strategy. Controlled experiments will be taken by dividing surgeons into two groups with one trained by the simulator and one without training to determine the benefits and drawbacks of our simulator. Further improving works are desired, including modeling the anatomic structures of Calot's triangle accurately, solving technical challenges related to realistic soft tissue simulation and integrating other training tasks for a better user experience.

\section{ACKNOWLEDGMENTS}

The authors appreciate the volunteers from National Center for Computer Animation (NCCA) and surgeons for their valuable comments and helpful suggestions. This research has been partly supported by Bournemouth University $\mathrm{PhD}$ scholarship and Hengdaoruyi Company.

\section{REFERENCES}

Medhat Alaker, Greg R Wynn, and Tan Arulampalam. 2016. Virtual reality training in laparoscopic surgery: a systematic review \& meta-analysis. International fournal of Surgery 29 (2016), 85-94.

Pamela B Andreatta, Eric Maslowski, Sean Petty, Woojin Shim, Michael Marsh, Theodore Hall, Susan Stern, and Jen Frankel. 2010. Virtual reality triage training provides a viable solution for disaster-preparedness. Academic emergency medicine 17, 8 (2010), 870-876.

David Baraff and Andrew Witkin. 1998. Large steps in cloth simulation. In Proceedings of the 25th annual conference on Computer graphics and interactive techniques. ACM, 43-54.

Jan Bender, Dan Koschier, Patrick Charrier, and Daniel Weber. 2014. Position-based simulation of continuous materials. Computers \& Graphics 44 (2014), 1-10.

Stuart Booth, FD Angelis, and Thore Schmidt-Tjarksen. 2003. The influence of changing haptic refresh-rate on subjective user experiences-lessons for effective touch-based applications. In Proceedings of eurohaptics. Citeseer, 374-383.
Sofien Bouaziz, Sebastian Martin, Tiantian Liu, Ladislav Kavan, and Mark Pauly. 2014. Projective dynamics: fusing constraint projections for fast simulation. ACM Transactions on Graphics (TOG) 33, 4 (2014), 154.

Murat Cenk Cavusoglu, Tolga G Goktekin, and Frank Tendick. 2006. GiPSi: a framework for open source/open architecture software development for organ-level surgical simulation. IEEE Transactions on Information Technology in Biomedicine 10, 2 (2006), 312-322.

Johan Creutzfeldt, Leif Hedman, Christopher Medin, Wm LeRoy Heinrichs, and Li Felländer-Tsai. 2010. Exploring virtual worlds for scenario-based repeated team training of cardiopulmonary resuscitation in medical students. fournal of medical Internet research 12, 3 (2010).

Gerald M Fried, Liane S Feldman, Melina C Vassiliou, Shannon A Fraser, Donna Stanbridge, Gabriela Ghitulescu, and Christopher G Andrew. 2004. Proving the value of simulation in laparoscopic surgery. Annals of surgery 240, 3 (2004), 518.

Anthony G Gallagher, E Matt Ritter, Howard Champion, Gerald Higgins, Marvin P Fried, Gerald Moses, C Daniel Smith, and Richard M Satava. 2005. Virtual reality simulation for the operating room: proficiency-based training as a paradigm shift in surgical skills training. Annals of surgery 241, 2 (2005), 364-372.

Will Goldstone. 2009. Unity game development essentials. Packt Publishing Ltd.

Maya M Hammoud, Francis S Nuthalapaty, Alice R Goepfert, Petra M Casey, Sandra Emmons, Eve L Espey, Joseph M Kaczmarczyk, Nadine T Katz, James J Neutens, and Edward G Peskin. 2008. To the point: medical education review of the role of simulators in surgical training. American fournal of Obstetrics \& Gynecology 199, 4 (2008), 338-343.

Mike Jackson, Steve Crouch, and Rob Baxter. 2011. Software evaluation: criteria-based assessment. Software Sustainability Institute (2011).

Keaton Jones. 2014. Laparoscopic cholecystectomy explained. "https://www.youtube. com/watch?v=a7rIFlvZM0I\&t=139s"

Youngjun Kim, Laehyun Kim, Deukhee Lee, Sangkyun Shin, Hyunchul Cho, Frédérick Roy, and Sehyung Park. 2015. Deformable mesh simulation for virtual laparoscopic cholecystectomy training. The Visual Computer 31, 4 (2015), 485-495.

James F Knight, Simon Carley, Bryan Tregunna, Steve Jarvis, Richard Smithies, Sara de Freitas, Ian Dunwell, and Kevin Mackway-Jones. 2010. Serious gaming technology in major incident triage training: a pragmatic controlled trial. Resuscitation 81, 9 (2010), 1175-1179.

Przemyslaw Korzeniowski. 2016. uFLex integrates NVidia Flex with Unity3D.

Sergei N Kurenov, William W Cance, Ben Noel, and David W Mozingo. 2009. Gamebased mass casualty burn training. Studies in health technology and informatics 142 (2009), 142-144.

Tiantian Liu, Adam W Bargteil, James F O'Brien, and Ladislav Kavan. 2013. Fast simulation of mass-spring systems. ACM Transactions on Graphics (TOG) 32, 6 (2013), 214.

Miles Macklin, Matthias Müller, Nuttapong Chentanez, and Tae-Yong Kim. 2014. Unified particle physics for real-time applications. ACM Transactions on Graphics (TOG) 33, 4 (2014), 153

Stefan Marks, JA Windsor, and Burkhard Wünsche. 2007a. Collaborative soft object manipulation for game engine-based virtual reality surgery simulators. (2007).

Stefan Marks, John Windsor, and Burkhard Wünsche. 2007b. Evaluation of game engines for simulated surgical training. In Proceedings of the 5th international conference on Computer graphics and interactive techniques in Australia and Southeast Asia. ACM, 273-280.

Matthias Müller, Bruno Heidelberger, Marcus Hennix, and John Ratcliff. 2007. Position based dynamics. Fournal of Visual Communication and Image Representation 18, 2 (2007), 109-118.

Matthias Müller, Bruno Heidelberger, Matthias Teschner, and Markus Gross. 2005. Meshless deformations based on shape matching. In ACM transactions on graphics (TOG), Vol. 24. ACM, 471-478.

Jun J Pan, Jian Chang, Xiaosong Yang, Hui Liang, Jian J Zhang, Tahseen Qureshi, Robert Howell, and Tamas Hickish. 2015. Virtual reality training and assessment in laparoscopic rectum surgery. The International fournal of Medical Robotics and Computer Assisted Surgery 11, 2 (2015), 194-209.

M. Poyade, M. Kargas, and V. Portela. (2014)-. Haptic Plug-In for Unity.

Kun Qian, Junxuan Bai, Xiaosong Yang, Junjun Pan, and Jianjun Zhang. 2015. Virtual reality based laparoscopic surgery simulation. In Proceedings of the 21st ACM Symposium on Virtual Reality Software and Technology. ACM, 69-78.

James C Rosser, Paul J Lynch, Laurie Cuddihy, Douglas A Gentile, Jonathan Klonsky, and Ronald Merrell. 2007. The impact of video games on training surgeons in the 21st century. Archives of surgery 142, 2 (2007), 181-186.

Eftychios Sifakis and Jernej Barbic. 2012. FEM simulation of 3D deformable solids: a practitioner's guide to theory, discretization and model reduction. In $A C M S I G$ GRAPH 2012 Courses. ACM, 20.

Tarja Susi, Mikael Johannesson, and Per Backlund. 2007. Serious games: An overview.

M Suzuki, S Akaishi, T Rikiyama, T Naitoh, MM Rahman, and S Matsuno. 2000. Laparoscopic cholecystectomy, Calot's triangle, and variations in cystic arterial supply. Surgical endoscopy 14, 2 (2000), 141-144.

Jinglu Zhang, Jian Chang, Xiaosong Yang, and Jian J Zhang. 2017. Virtual Reality Surgery Simulation: A Survey on Patient Specific Solution. In International Workshop on Next Generation Computer Animation Techniques. Springer, 220-233. 
Olek C Zienkiewicz and Robert L Taylor. 2005. The finite element method for solid and structural mechanics. Butterworth-heinemann. 